\title{
VISIÓN Y OLFATO EN LAS EVALUACIONES DE ATRACTIVO QUE HACEN HOMBRES HOMOSEXUALES
}

\author{
SmeLl AND Sight ON THE ATtRACtION FELt by hOMOSEXUAL MEN
}

\author{
José María Jiménez Orvañanos*, Irvin Antonio Camacho Delgado**, \\ Rocío Alcázar Gaspar*** Y JOAQUINA PALOMAR ${ }^{* \star * *}$
}

\author{
*Estudiante de Psicología de la Universidad Iberoamericana (UIA). \\ E-Mail: chema.jimorv@gmail.com \\ ${ }^{* \star E}$ Estudiante de Psicología de la Universidad Iberoamericana (UIA). E-Mail: irvin.jap@gmail.com \\ ${ }^{* * *}$ Estudiante de Psicología de la Universidad Iberoamericana (UIA). \\ ****Doctora en Psicología. Profesor-Investigador de la Universidad Iberoamericana (UIA). \\ E-Mail: joaquina.palomar@ibero.mx \\ Universidad Iberoamericana (UIA). Arroyo de los Ahuehuetes 30, México D.F., México.
}

\section{RESUMEN}

Las investigaciones sobre el papel de los sentidos en la elección de pareja que hacen hombres y mujeres han confirmado la presencia de procesos a nivel neurofisiológico cuya influencia va más allá de las decisiones conscientes que pudieran tomarse en cuanto a la pareja con la que deciden estar. Sin embargo, la mayoría de las investigaciones realizadas hasta ahora tienen un enfoque en parejas heterosexuales y son pocos los estudios que examinan el papel de los sentidos en la elección de pareja que hacen las personas homosexuales.

El objetivo del estudio realizado fue evaluar la importancia y la relación que tienen el olfato y la visión en la atracción de hombres homosexuales hacia hombres, tanto hetero como homosexuales. Un grupo de 14 hombres homosexuales evaluó el atractivo de fotografías y camisetas (remera de algodón de mangas cortas) provenientes de hombres hetero y homosexuales. Los resultados indicaron que el olor de las camisetas utilizadas por hombres homosexuales resultó significativamente más atractivo que el olor de las camisetas utilizadas por hombres heterosexuales en las evaluaciones realizadas. Por otra parte, se realizaron análisis de regresión múltiple y sus resultados mostraron que el atractivo del olor de las camisetas utilizadas por hombres homosexuales predice significativamente el atractivo global de estos hombres. Estos resultados sugieren la presencia de una capacidad en hombres homosexuales para distinguir inconscientemente a hombres heterosexuales de hombres homosexuales a partir de estímulos olfativos, lo que a su vez apunta a una constitución neurofisiológica en particular, invitando a realizar más investigaciones en el tema.

Palabras clave: Homosexuales; Olfato; Visión; Atractivo; Pareja; Feromonas.

\section{ABSTRACT}

Previous works about the role of the senses in mating choices made by men and women have confirmed the existence of neurophysiologic processes which influence our decisions about whom we'd like to have a relationship with, well beyond our conscious capacity to select.

Most of these works, however, are developed from a focus on Evolutionary Psychology, whose 
theoretical axis is the transmission of genes from one generation to another by means of reproduction. Therefore, they have centered exclusively on the evaluations of attractiveness made by heterosexual people, thus disregarding the role of the senses in the mating choices of their homosexual counterparts.

Our goal in the present study was to evaluate the relationship between smell and sight, as well as their influence on the attraction felt by homosexual men towards heterosexual and homosexual men.

A group of 14 homosexual men assessed the attractiveness of a series of stimuli that came from homosexual and heterosexual males. Subjects ranged from 16 to 26 years of age $(M=21.21 ; S D=2.6)$, participated voluntarily and were selected through accidental, non probability sampling. All of them were recruited in a meeting of the Jóvenes LGBT México Group, thanks to the support of one of its coordinators, with the objective of accommodating the schedules of the population under study.

The stimuli consisted of facial pictures of homosexual and heterosexual men, as well as cotton tee shirts previously worn by the same subjects. All stimuli were collected from 10 men between 21 and 25 years of age $(M=22.3 ; S D=1.49)$, acquaintances of the researchers. Invitations were sent via e-mail. Participants were informed that the objective of the study was to assess the importance of the senses in the mating choices of human males.

Findings may be summarized as follows: the olfactory attractiveness of the tee shirts and the visual attractiveness of the pictures did not correlate for either of the two groups; only the olfactory attractiveness of the tee shirts differed significantly depending on the sexual orientation of the wearers; both the visual attractiveness of the pictures and the olfactory attractiveness of the tee shirts predicted the global attractiveness of heterosexual subjects, though the visual attractiveness of the pictures did so more significantly; and, finally, only the olfactory attractiveness of the tee shirts predicted the global attractiveness of homosexual subjects.

In other words, the smell of the tee shirts worn by homosexual men resulted significantly more attractive to the homosexual evaluators than did the smell of the tee shirts worn by heterosexual men. On the other hand, only the attractiveness of the smell of the tee shirts worn by homosexual men predicted significantly the global attractiveness of those men.

This suggests that the homosexual men who participated as evaluators were able to distinguish, without being conscious of it, the sexual orientation of the men who wore the shirts, parting from their olfactory attractiveness.

The most important implication of our findings is that homosexual people may function in a different neurophysiologic manner than their heterosexual counterparts, at least in regards to the influence of sensory perception on the mating choices they make. In particular, that factors independent from reproduction may have a role in human mating choices. Therefore, in future research conducted on the matter, the sexual orientation of the subjects should be considered, additionally to their biological sex.

Key words: Homosexual; Smell; Vision; Attraction; Mating; Couple; Pheromones.

\section{INTRODUCCIÓN}

La investigación sobre el papel de los sentidos en la elección de pareja se ha basado en gran medida, en la Psicología Evolucionista. Dicha postura considera que las características de un organismo se modifican en relación a su entorno con tal de buscar garantizar su éxito reproductivo (Thornhill \& Gangestad, 2003).

A partir de este planteo teórico, el interés de los estudios se ha centrado en la elección heterosexual de pareja, ya que ésta conduce a la transmisión de genes de una generación a otra, así como a la continuidad de la especie humana. En particular, se han descrito con más detalle los mecanismos que operan en los juicios de atractivo que hacen mujeres heterosexuales. Esto quizá se explique por el hecho de que sus elecciones de pareja suelen implicar más riesgo debido a los costos que 
les impone la reproducción (Thornhill \& Gangestad, 2003).

Esto último da lugar a considerables lagunas con respecto al conocimiento sobre las estrategias de selección de pareja que usan los hombres, más aún los hombres homosexuales. Dado que la interacción sexual entre sujetos de ciertos grupos no puede explicarse en términos evolucionistas, se considera que es necesario profundizar en el conocimiento que se tiene sobre el tema.

Las personas dan señales de sus cualidades hereditarias a parejas potenciales. Algunas de estas claves son físicas tales como la simetría de cara y cuerpo, las formas masculinas o femeninas de la cara y la figura corporal, y son percibidas con la visión, el tacto y el oído. Otras, como el olor corporal, son químicas y se detectan a través del olfato (Cerda, Mondragón \& Díaz, 2000; Cornwell et al., 2004).

\section{OLOR CORPORAL}

Existe evidencia de que los olores corporales individualmente únicos (huellas olfativas) son regulados por el antígeno humano de leucocitos (HLA), una serie de genes encargados del sistema inmune (Jacob, Mc Clintock, Zelano \& Ober, 2002). El olor producido por las glándulas axilares de un individuo puede dar información sobre las características de su sistema inmune (Sergeant, Dickins, Davies \& Griffiths, 2007). Además, se cree que las huellas olfativas sirven para que los humanos distingamos a otras personas como parte de tal o cual subgrupo social (Martins et al., 2005).

Se ha observado que los sujetos describen olores corporales como más agradables cuando provienen de personas que tienen una cantidad intermedia de alelos (formas alternativas de un gen) del antígeno humano de leucocitos correspondiente a los suyos. Dichos olores resultan menos placenteros cuando pertenecen a individuos cuyos antígenos humanos de leucocitos son absolutamente iguales a los del evaluador o cuando son completamente distintos. Esto puede deberse a un mecanismo evolutivo para preservar la inmuno-competencia de las crías es decir, maximizar su protección contra enfermedades, como así también reducir al mínimo las mutaciones genéticas (Levin, 2004; Martins et al., 2005).

En el caso de las mujeres, el olor axilar transmite información sobre su estado de fertilidad y resulta más agradable para los hombres durante la fase folicular, cuando la probabilidad de concepción es alta, que en la fase lútea, después de la ovulación (Havlíček, Dvořákov, Barto \& Flegr, 2006; Shoup, Streeter \& McBurney, 2008).

También se ha observado que el olor corporal tiene una gran influencia sobre el comportamiento en pareja y sobre la satisfacción sexual de las mujeres (GarverApgar, Gangestad, Thornhill, Miller \& Olp, 2006). Mientras más alelos del antígeno humano de leucocitos compartían con sus parejas hombres, un grupo de participantes mujeres reportó con más frecuencia ser infieles, así como experimentar mayor atracción por otros hombres, en especial durante sus días fértiles. Lo anterior se debe a que las parejas extramaritales son compatibles genéticamente en cuanto a la similitud intermedia de alelos. Lo anterior, sin embargo, no predecía la satisfacción con respecto a su relación de pareja, sino sólo su satisfacción sexual.

Los hombres que participaron en el mismo estudio no reportaron diferencias en la capacidad de respuesta sexual en relación con las similitudes entre los alelos del antígeno humano de leucocitos. Los sujetos tampoco reportaron tener relaciones extramaritales. Sin embargo, sí reportaron percibir menos satisfecha a su pareja en el aspecto sexual (Garver-Apgar et al., 2006).

\section{ATRACTIVO FACIAL}

La mayoría de los investigadores concuerdan en que el atractivo facial de una persona se debe a qué tanto se asemeja su rostro al promedio, qué tan simétrico es y a ciertos rasgos masculinos o femeninos. Las 
pruebas directas realizadas para comprobar esta hipótesis muestran que el grado de parecido de un rostro al promedio es suficiente para predecir su atractivo, sin importar su grado de simetría (DeBruine, Jones, Unger, Little \& Feinberg, 2007; Rennels, Bronstad \& Langlois, 2008). La masculinidad es el rasgo que mejor predice el atractivo en un rostro de hombre. Sin embargo, también se encontró que los extremos en masculinidad no son agradables (Rennels et al., 2008).

En un experimento (DeBruine et al., 2007) se observó que la adaptación a ciertas características faciales altera los juicios que se hacen sobre el atractivo de rostros de mujeres. El tipo de cara al que una población está habituada (rostro prototipo) influye sobre sus evaluaciones faciales. Por ejemplo, la exposición a muchas caras redondas cambia el prototipo de un sujeto a caras igualmente redondas, lo cual recalibra el valor a cero para este tipo de rostros (DeBruine et al., 2007). Lo anterior es una consideración importante al trabajar con distintos grupos que podrían estar habituados a tipos particulares de rostros.

De forma similar a lo que sucede con el olor corporal, las mujeres son más atractivas para los hombres a lo largo de la fase folicular de su ciclo menstrual, que durante la fase lútea del mismo. Se ha observado que estos cambios en el atractivo visual se deben a modificaciones en los niveles de liberación hormonal, que a su vez influyen sobre el olor corporal (Roberts et al., 2004).

\section{VISIÓN Y OLFATO}

Ciertos estudios muestran que el olor es de especial relevancia para las mujeres que eligen un amante, mientras que la visión juega el papel principal en las evaluaciones de este tipo realizadas por hombres (Herz \& Inzlicht, 2002; Sergeant, Davies, Dickins \& Griffiths, 2005). Sin embargo, Foster (2008) advirtió que estos hallazgos podrían deberse al uso de auto-reportes.

Existe una relación clara entre el olor y la memoria. Se sabe que el primero desenca- dena recuerdos autobiográficos, así como que se asocia con los sentimientos (Mc Burney, Shoup \& Streeter, 2006). Desde esta lógica, es coherente considerar que el olfato sea relevante para la elección de pareja. Pero según Foster (2008), cuando la evaluación se hace al momento de percibir un estímulo, con menor interferencia de la memoria, el olor no resulta tan importante.

Foster (2008) propuso desde una perspectiva evolucionista que sería más razonable que los humanos recurrieran a su sentido más desarrollado (la vista) al elegir a sus parejas para garantizar la continuidad de su especie. En un experimento que realizó, el atractivo facial de los hombres participantes predijo su atractivo global de forma significativa. Los resultados sugirieron que cuando las mujeres hacen juicios de atractivo en hombres, las claves visuales son significativamente más importantes que las olfativas.

En contraste, deben considerarse los resultados de un estudio que analizó muestras del ADN de parejas en busca de similitudes en sus genes del antígeno humano de leucocitos (Chaix, Cao \& Donnelly, 2008). Los autores concluyeron que es más probable encontrar mecanismos olfativos de selección cuando no hay otros factores que influyan sobre la elección de pareja (en este caso, grupos endógenos donde el aspecto facial no variaba mucho entre sujetos).

Paralelamente a estos hallazgos, se ha sugerido que los humanos consideramos más de una señal sexual a la vez, ya que así se reducen los errores al evaluar a parejas potenciales. Por ejemplo, las mujeres prefieren olores corporales de hombres con altos grados de simetría bilateral en comparación con hombres más asimétricos (Cornwell et al., 2004; Herz \& Inzlicht, 2002). Esta simetría bilateral se refiere a la distribución equitativa de las funciones de los hemisferios cerebrales y puede percibirse en la configuración de los rostros (Thornhill \& Gangestad, 2003). Su contrario, conocido como asimetría fluctuante, se refiere a personas en las que dichas funciones se distribuyen de forma ligeramente desigual entre los hemisferios debido a perturbaciones genéticas o am- 
bientales; también, en teoría, es perceptible en el rostro de los hombres (Thornhill \& Gangestad, 2003).

Una baja asimetría fluctuante sugiere una condición fenotípica sana (el fenotipo es la expresión visible de un rasgo determinado por un gen, por ejemplo: ojos azules, verdes o cafés), así como una alta calidad genética. Los hombres con baja asimetría fluctuante tienen cuerpos más grandes, son más musculosos, vigorosos y tienden menos al sometimiento social. Están más dispuestos a competir directamente con otros hombres, sus rostros tienden a ser masculinos y sus coeficientes intelectuales a ser más elevados (Thornhill \& Gangestad, 2003).

\section{SUJETOS HOMOSEXUALES}

\section{OLOR CORPORAL}

La evidencia sugiere que las mujeres y los hombres homosexuales podrían producir olores axilares que los distinguen de los heterosexuales, así como que los hombres homosexuales perciben los olores de forma distinta que los heterosexuales (Martins et al., 2005). La explicación teórica de estos hallazgos remite a la hipótesis neurohormonal de la orientación sexual, según la cual la presencia de hormonas adrenales durante el desarrollo prenatal potencia la atracción hacia mujeres, mientras que la ausencia de dicha actividad facilita la atracción por hombres (Mustanski, Chivers \& Bailey, 2002).

Savic, Berglund y Lindström (2005) reportaron un patrón de activación hipotalámica ante feromonas humanas distinto para diferentes orientaciones sexuales. Las feromonas son mensajeros químicos, indetectables por la conciencia, que inducen cambios fisiológicos o conductuales en el comportamiento de otros individuos. Son producidas sobre todo por las glándulas apócrinas en las regiones axilares y púbicas y aparecen en mayor número en mujeres que en hombres (Cerda et al., 2000; Grammer, Fink \& Neave, 2005). Tanto los hombres homosexuales como las mujeres heterosexuales y a diferencia de los hombres heterosexuales, prefieren el olor de feromonas masculinas (Savic et al., 2005).

En otro estudio, un grupo de mujeres heterosexuales calificó el olor de hombres homosexuales como significativamente más agradable, sexy y preferible que el de hombres heterosexuales. Sin embargo, la magnitud de las diferencias fue pequeña. Más aún, el olor de hombres homosexuales fue calificado neutralmente en las escalas utilizadas. Desde esta óptica, podría decirse que dicho olor fue menos desagradable que los olores de hombres heterosexuales, pero no particularmente agradable en sí mismo (Sergeant et al., 2007).

Por último, Martins y colaboradores (2005) observaron que los hombres homosexuales prefieren el olor de sus pares homosexuales y de las mujeres heterosexuales. Además, advirtieron que existe una equivalencia entre los juicios de hombres y mujeres heterosexuales: ambos prefieren olores de personas heterosexuales sobre olores de sujetos homosexuales. También prefieren el olor de mujeres homosexuales sobre el olor de hombres homosexuales. Finalmente, las mujeres homosexuales prefieren los olores de sujetos heterosexuales en general, así como el olor de mujeres homosexuales antes que el de hombres homosexuales.

\section{Atractivo facial}

La corteza orbitofrontal (segmento de la corteza prefrontal) es en parte responsable de representar cuán placenteros resultan los estímulos sensoriales, entre ellos, rostros hermosos $\mathrm{u}$ otros reforzadores positivos o negativos. Se ha observado que la belleza facial suscita respuestas en el sistema cerebral de recompensa, una conexión entre el área ventral del mesencéfalo y el núcleo accumbens, principal productor de dopamina. Se cree que esta vía regula el comportamiento a través de las sensaciones placenteras (Kranz \& Ishai, 2006).

En un trabajo de Kranz e Ishai (2006) con imágenes obtenidas con resonancia magné- 
tica funcional se encontró que los rostros de hombre activaban la corteza orbitofrontal y el núcleo mediodorsal del tálamo en mujeres heterosexuales y en hombres homosexuales, mientras que las caras de mujer tenían este efecto en mujeres homosexuales y en hombres heterosexuales.

Cuando sólo se consideraba el género y no la orientación sexual, no había una diferencia significativa en la activación de las regiones mencionadas. Esto sugiere que la respuesta a los rostros fue modulada por la preferencia sexual y no por el género. Los resultados hacen pensar que la corteza orbitofrontal representa valores de recompensa para los rostros de parejas sexuales potenciales, incluyendo individuos del mismo sexo, sin tener en cuenta la reproducción.

\section{Objetivos}

El objetivo del estudio realizado es contribuir al conocimiento actual sobre las señales sexuales en la elección de pareja de hombres homosexuales, cuyos juicios de atractivo (como se ha demostrado en estudios recientes) podrían funcionar de forma distinta que los de sus contrapartes heterosexuales.

En esta línea, el interés fue explorar la relación que existe entre el tipo de señal sexual (olfativa o visual) y el juicio de atractivo que se hace sobre una potencial pareja.

A partir de la siguiente pregunta de investigación: ¿tienen más importancia los estímulos olfativos o los visuales en las evaluaciones de atractivo que hacen hombres homosexuales?, la idea fue que un grupo de hombres homosexuales evaluara el atractivo de una serie de estímulos provenientes de otros hombres, tanto homo como heterosexuales. Los estímulos fueron de dos tipos: olfativos y visuales.

\section{Metodología}

Se trató de un diseño cuasi-experimental, ya que los sujetos no fueron clasificados en grupos de forma aleatoria y no existió un grupo control, pero se manipularon las variables independientes y transversal, en vista de que la recolección de datos se hizo en un único momento.

\section{VARIABLES}

La variable dependiente fue el Atractivo global de los estímulos, que consistió en el atractivo percibido por los evaluadores al presentárseles simultáneamente una camiseta y una fotografía provenientes del mismo sujeto, fuera éste homo o heterosexual.

Las variables independientes fueron las siguientes:

1.- Estímulos olfativos: una serie de camisetas usadas por hombres homo y heterosexuales.

2.- Estímulos visuales: una serie de fotografías del rostro de hombres homo y heterosexuales.

3.- Orientación sexual de los hombres evaluados.

\section{PARTicipantes}

Mediante un muestreo no probabilístico accidental, se solicitó la participación voluntaria de 14 hombres homosexuales de 16 a 26 años $(M=21.21, D E=2.6)$ que concurrían a una reunión del Grupo Jóvenes LGBT México, gracias al apoyo de uno de sus coordinadores.

Se aplicó la Escala Kinsey de 7 puntos (Kinsey, Pomeroy \& Martin, 1948) a todos los participantes que aceptaron la invitación, con el fin de corroborar su orientación sexual antes de que evaluaran los estímulos.

Ocho de ellos eligieron el puntaje 6 en dicha escala (exclusivamente homosexual), mientras que los 6 restantes escogieron el 5 (predominantemente homosexual y sólo incidentalmente heterosexual). Diez eran estudiantes y 4 trabajadores asalariados. Cua- 
tro de los primeros cursaban el bachillerato y los otros 6, la licenciatura.

\section{ESTíMULOS}

Los estímulos se obtuvieron de 10 hombres de 21 a 25 años $(M=22.3, D E=1.49)$, cuya participación se solicitó mediante un muestreo no probabilístico accidental. Se enviaron invitaciones a los sujetos vía correo electrónico. Se les informó que el objetivo de la investigación era estudiar la importancia de los sentidos (olfato y visión) en la elección de pareja que hacen los hombres.

Los estímulos fueron de dos tipos: visuales y olfativos. Los estímulos visuales fueron fotografías del rostro de los participantes. Las mismas se tomaron a los sujetos individualmente con fondo blanco. Se les pidió que retiraran, en la medida de lo posible, el pelo de su rostro, así como que se quitaran aretes, gafas o cualquier otro accesorio o maquillaje. Las fotografías se tomaron alrededor de una semana antes de la aplicación, en distintos momentos del día y dicho material fue entregado a los participantes. Para disminuir posibles efectos de la vestimenta y del cuerpo, se recortó la imagen digitalmente de forma que sólo fuera visible el rostro (de la manzana de Adán hacia arriba). Todas las fotografías eran en blanco y negro y fueron editadas digitalmente para que tuvieran calidades similares en cuanto a brillo y contraste.

Los estímulos olfativos consistieron en camisetas blancas de algodón usadas por los sujetos antes de la aplicación. Cuatro días antes del experimento se les entregó a los sujetos una camiseta blanca de algodón, lavada previamente con detergente sin olor, también se les dio una bolsa de plástico que se podía sellar herméticamente y un jabón neutro sin aroma. La consigna fue que usaran la camiseta mientras dormían durante dos noches consecutivas. Después del primer período de uso, debían guardarla en la bolsa, extraerla de nuevo la segunda noche, usarla, guardarla y finalmente, entregarla a los investigadores un día después.
Se proporcionaron las siguientes instrucciones:

1.- Bañarse con un jabón neutro por las noches, antes de usar las camisetas.

2.- No usar productos higiénicos con aroma (desodorante, crema de afeitar, loción) durante las noches.

3.- Evitar los siguientes alimentos desde 2 días antes del período de uso de las camisetas: apio, pepperoni, cebolla, hierbas o especies con olores ásperos o picantes (curry, paprika), chile verde, quesos fuertes, col, yogurt, espárragos y cordero.

4.- No beber alcohol ni usar drogas recreacionales al usar la camiseta.

5.- No fumar mientras tenían puesta la camiseta.

6.- No tener relaciones sexuales mientras usaban la camiseta.

7.- No dormir con otras personas al usar la camiseta.

Los sujetos usaron las camisetas durante las noches del miércoles y del jueves de la misma semana y las entregaron a los investigadores el viernes por la tarde.

Los experimentadores refrigeraron las camisetas a partir de ese momento hasta la mañana del domingo, un par de horas antes de iniciar la aplicación. Se procedió de esta forma debido a la disponibilidad del Grupo Jóvenes LGBT para participar en la investigación. En vista de que ellos se reunían los domingos, hubo que asignar las noches cuando los otros sujetos usarían las camisetas en relación con eso.

Los investigadores consideraron prudente que los sujetos usaran las camisetas durante el miércoles y el jueves, ya que haberlas usado durante la noche del viernes hubiera aumentado la posibilidad de variancia de error al tornar más difícil que los sujetos respetaran todas las instrucciones. 
Tanto los estímulos visuales como los olfativos se clasificaron en dos grupos: estímulos provenientes de hombres homosexuales y estímulos provenientes de hombres heterosexuales.

Dicha clasificación se hizo en base a los resultados obtenidos con la administración de la Escala Kinsey de 7 puntos.

\section{INSTRUMENTOS}

- Se administró la Escala Kinsey de 7 puntos (Kinsey et al., 1948) traducida al español por los investigadores. Dicho instrumento describe la orientación sexual como un continuo, más que como una categoría estática y excluyente de otras orientaciones sexuales. Los sujetos responden con una escala de 0 a 6 , en la que cero corresponde a exclusivamente heterosexual, 6 a exclusivamente homosexual y el punto medio, a bisexual. También existe la posibilidad de responder ' $X$ ', que significa asexual o sin sexualidad.

El instrumento se basa en los Reportes Kinsey sobre el comportamiento sexual en hombres y mujeres estadounidenses. No es una prueba psicométrica, por lo cual no se cuenta con índices de confiabilidad ni de validez.

Cinco de los 10 sujetos respondieron 0 (exclusivamente heterosexual), 2 eligieron la opción 5 (predominantemente homosexual, sólo incidentalmente heterosexual) y 3, la opción 6 (exclusivamente homosexual).

Los estímulos de los sujetos que respondieron 0 se asignaron al grupo de estímulos provenientes de hombres heterosexuales, mientras que los de los sujetos que respondieron 5 ó 6 se colocaron en un solo grupo, denominado estímulos provenientes de hombres homosexuales.

- Se utilizaron escalas Likert de 11 puntos, diseñadas por los experimentadores: una para evaluar el Atractivo Visual de las fotografías, otra para el Atractivo Olfativo de las camisetas y una más para el
Atractivo Global de los estímulos. Las escalas iban de 0 a 10 , donde 0 significaba nada atractivo y 10 quería decir extremadamente atractivo.

\section{PROCEDIMIENTO}

Las sesiones de evaluación se dividieron en tres fases sucesivas: una para los estímulos olfativos, otra para los visuales y una última para estímulos olfativos y visuales combinados (Atractivo Global).

Los estímulos olfativos fueron representados con las camisetas blancas de algodón, previamente usadas, dobladas dentro de bolsas herméticamente selladas.

Los estímulos visuales fueron las fotografías impresas de $10 \times 15 \mathrm{~cm}$ del rostro de los sujetos a evaluar y fueron presentadas boca abajo.

Para la fase de Atractivo Global, se presentaban las camisetas dentro de las bolsas simultáneamente con las fotografías boca abajo.

Cada sesión se condujo en una habitación de la casa donde se reúne el Grupo Jóvenes LGBT México. La habitación estaba en la parte trasera, por lo cual se encontraba relativamente aislada de ruido y de olores del ambiente (sólo los investigadores y los participantes tuvieron acceso a la sala). Era un pequeño estudio con una mesa larga en el centro. Con cuatro divisiones hechas de cartón, se formaron cinco cubículos sobre la mesa. En cada fase se colocó un estímulo por cubículo, ya sean las camisetas para evaluar el atractivo olfativo, las fotografías para evaluar el atractivo visual o ambos estímulos apareados para evaluar su atractivo global.

El orden de presentación de cada serie fue determinado por el uso de tablas de números aleatorios, para evitar que los evaluadores asociaran el orden en que se presentarían las camisetas con el de las fotografías y así, asociaran cierta camiseta con una fotografía que no le correspondiera a partir de sus propias hipótesis.

Se invitaba a los evaluadores a participar en el experimento in situ. Se les explicaba que se estaba realizando un experimento, su 
propósito y en qué consistiría su participación. A quienes accedieron para participar, se les entregó un cuestionario en el que debían contestar la Escala Kinsey e informar su edad y ocupación. Ninguno de ellos contestó menos de cinco puntos de la Escala Kinsey. A continuación, se les entregó el formato de evaluación ${ }^{1}$ y se dieron las instrucciones para participar en el experimento.

Las instrucciones de evaluación fueron específicas para cada fase. Para la primera (Atractivo Olfativo), los participantes debían tomar la bolsa y abrirla para oler la camiseta. Para esto, podían sacarla un poco para olerla mejor si lo consideraban necesario, pero no podían tocar la camiseta directamente con las manos ni sacarla completamente de su bolsa. Al terminar de olerla, tenían que cerrar la bolsa, colocarla nuevamente en la mesa, anotar la calificación del atractivo en el formato de evaluación y continuar con el siguiente cubículo para repetir la operación hasta terminar de evaluar todas las camisetas.

Para evaluar las fotografías, los evaluadores tenían que voltear la fotografía para ver el retrato, devolver la fotografía a su posición original y anotar la calificación para poder proceder a evaluar la siguiente fotografía.

Finalmente, para evaluar el Atractivo Global, debían voltear la fotografía y, mientras la veían, tomar la bolsa con la camiseta, abrirla y olerla. Después de cerrar la bolsa y de regresar la fotografía a su posición original, anotaban la calificación en el formato de evaluación. A continuación repetían el mismo procedimiento con el resto de los estímulos.

Los participantes evaluaron los estímulos en un grupo de cuatro y posteriormente, en dos de cinco, conforme los investigadores conseguían su consentimiento para participar en el estudio. Dos sujetos evaluaron individualmente.

En vista de que sólo se contaba con cinco cubículos sobre la mesa, sólo se podían pre-

\footnotetext{
${ }^{1}$ Los protocolos pueden ser solicitados a los autores.
}

sentar cinco estímulos por vez. Por lo tanto, cada una de las fases tuvo que dividirse en dos partes. Así, por ejemplo, para la fase olfativa se colocaron cinco camisetas sobre la mesa, una por cubículo (en orden aleatorio). Los evaluadores pasaban a evaluarlas y al terminar, salían de la habitación. Entonces los experimentadores retiraban las camisetas evaluadas, colocaban las cinco camisetas restantes sobre la mesa, una por cubículo y al terminar de hacerlo, llamaban a los participantes para que continuaran con sus evaluaciones.

Antes de comenzar cada fase, los sujetos se reunían en un patio aledaño a la habitación donde se condujo el experimento, separado de la misma por un pasillo angosto. Desde ahí, de a uno ingresaban a la habitación. Al entrar, el primer participante pasaba al cubículo 1, evaluaba el estímulo que ahí se encontraba y al terminar, seguía con el estímulo del cubículo 2. Entonces, ingresaba el siguiente participante para evaluar el estímulo del cubículo 1, el cual acababa de desocuparse. Se procedió de esta manera hasta que todos los evaluadores del grupo terminaron de evaluar los estímulos de los 5 cubículos.

Cuando cada sujeto terminaba con los cinco estímulos, regresaba al patio. Durante este lapso de tiempo entre ambas fases, los evaluadores eran libres de comentar su experiencia si así lo deseaban. Los sujetos no tuvieron límite de tiempo para evaluar, aunque se observó que les tomaba entre 30 y 60 segundos por estímulo.

Durante las evaluaciones del Atractivo Visual, dos de los evaluadores comentaron a los investigadores que conocían a uno de los sujetos de las fotografías.

Por errores con el formato de evaluación se retiraron a dos sujetos de la muestra final.

\section{Resultados}

\section{Correlaciones de Pearson}

La primera hipótesis fue que habría una correlación positiva entre el Atractivo Visual 
de las fotografías de los sujetos homosexuales y el Atractivo Olfativo de las camisetas de los mismos sujetos. Para comprobarla, se realizó una prueba de correlación de Pearson. Se apareó el Atractivo Visual de las fotografías de los 5 evaluados heterosexuales con el Atractivo Olfativo de las camisetas de los mismos sujetos. De las 5 correlaciones significativas posibles, sólo se encontró una. En otras palabras, sólo en el caso de uno de los 5 hombres heterosexuales se encontró una correlación estadísticamente significativa $[r(13)=.533, p<.05]$. Esto sugiere que el Atractivo Visual de la fotografía de ese sujeto tuvo una relación con el Atractivo Olfativo de la camiseta que usó él. Sin embargo, en vista de que en ninguno de los otros cuatro casos se encontró una correlación estadísticamente significativa entre los mismos atractivos, no se considera que la correlación significativa encontrada sea un resultado generalizable.

La segunda hipótesis fue que habría una correlación positiva entre el Atractivo Visual de las fotografías de los sujetos heterosexuales y el Atractivo Olfativo de sus camisetas. Para ella, también se realizó una prueba de correlación de Pearson. Se apareó el Atractivo Visual de las fotografías de los cinco evaluados homosexuales con el Atractivo Olfativo de las camisetas que usaron los mismos sujetos. De las cinco posibles correlaciones significativas, sólo se encontró una estadísticamente significativa $[r(13)=.598$, $p<.05]$. Esto sugiere que el Atractivo Visual de su fotografía tuvo una relación con el Atractivo Olfativo de su camiseta. Sin embargo, en vista de que en ninguno de los otros cuatro casos se encontró una correlación estadísticamente significativa, tampoco se considera que la correlación significativa encontrada sea un resultado generalizable.

A partir de estos hallazgos, como sólo se encontraron dos de las 10 correlaciones significativas posibles, se puede decir que no se rechazaron las dos hipótesis nulas: (a) No se encontraron correlaciones significativas entre el Atractivo Olfativo de las camisetas y el Atractivo Visual de las fotografías para la mayoría de los evaluados heterosexuales. (b) No se encontraron correlaciones significativas entre el Atractivo Olfativo de las camisetas y el Atractivo Visual de las fotografías para la mayoría de los evaluados homosexuales.

Por lo tanto, no podría decirse que el Atractivo Visual se correlaciona, en la mayoría de los casos, con el Atractivo Olfativo de las camisetas, en ninguna de las dos orientaciones sexuales.

\section{Pruebas de WiLCOXON}

Para la tercera hipótesis (el Atractivo Visual de las fotografías diferiría significativamente a partir de la orientación sexual de los sujetos fotografiados), se realizó la Prueba de Wilcoxon. Se comparó el Atractivo Visual de las fotografías de los sujetos homosexuales con el Atractivo Visual de las fotografías de los sujetos heterosexuales. No se encontraron diferencias estadísticamente significativas entre los grupos. No se rechazó la hipótesis nula.

Con respecto a la hipótesis cuatro (que el Atractivo Olfativo de las camisetas diferiría significativamente a partir de la orientación sexual de los sujetos que las usaron), también se realizó una Prueba de Wilcoxon. Se contrastó el Atractivo Olfativo de las camisetas de los sujetos heterosexuales con el Atractivo Olfativo de las camisetas de los sujetos homosexuales. En este caso, sí se encontraron diferencias significativas $(z=-2.001, p<.05)$. El Atractivo Olfativo de las camisetas de los sujetos homosexuales fue distinto del Atractivo Olfativo de las camisetas de los sujetos heterosexuales, en un sentido negativo. La media del Atractivo Olfativo de las camisetas de los sujetos homosexuales fue superior a la media del mismo atractivo de las camisetas de los sujetos heterosexuales. En otras palabras, el olor de las camisetas de los sujetos homosexuales resultó significativamente más atractivo para los evaluadores que el olor de las camisetas de los sujetos heterosexuales. En el caso de la hipótesis cuatro, se rechazó la hipótesis nula. 
Por último, se realizó la Prueba de Wilcoxon al trabajar con la hipótesis cinco: el Atractivo Global diferiría significativamente a partir de la orientación sexual de los sujetos evaluados. No se encontraron diferencias estadísticamente significativas entre el Atractivo Global de los sujetos homosexuales y el Atractivo Global de los sujetos heterosexuales. Entonces, no se rechazó la hipótesis nula.

\section{REGRESIONES MÚLTIPLES}

Se hizo un análisis de regresión múltiple para la hipótesis seis: el Atractivo Olfativo de las camisetas de los sujetos homosexuales predecirá de forma estadísticamente más significativa, en comparación con el atractivo de sus fotografías, el Atractivo Global de los mismos sujetos. Primero, se encontraron correlaciones significativas entre el Atractivo Visual de las fotografías de los sujetos heterosexuales y el Atractivo Global de los mismos sujetos $[r(13)=.703, p<.01]$, así como entre el Atractivo Olfativo de las camisetas de los sujetos heterosexuales y el Atractivo Global de los mismos sujetos $[r(13)=.592, p<.05]$. Por lo tanto, se continuó con la regresión. Obsérvese que la correlación entre el Atractivo Visual de las fotografías y el Atractivo Global fue más fuerte y significativa que la correlación entre el Atractivo Olfativo de las camisetas y el Atractivo Global. Los resultados de la regresión se presentan en la Tabla 1 .

Ambas variables predijeron significativamente el Atractivo Global de los estímulos, y juntas, explicaron una porción considerable de la variancia del Atractivo Global de los estímulos $\left[R^{2}=.682, F(1\right.$, $12)=11.77, p<.01]$. Sin embargo, el Atractivo Visual de las fotografías lo hizo más significativamene que el Atractivo Olfativo de las camisetas.

En el caso de la séptima hipótesis (el Atractivo Visual de las fotografías de los sujetos heterosexuales predeciría de forma estadísticamente más significativa, en comparación con el Atractivo Olfativo de sus ca- misetas, el Atractivo Global de los mismos sujetos), también se realizó un análisis de regresión múltiple. Se encontraron correlaciones significativas entre el Atractivo Olfativo de los sujetos homosexuales y el Atractivo Global de los mismos sujetos $[r(13)=.813$, $p=000]$, así como entre el Atractivo Visual de las fotografías y el Atractivo Global del mismo grupo $[r(13)=.671, p<.01]$. Luego se continuó con la regresión.

De forma contraria a lo ocurrido con los estímulos de los sujetos heterosexuales, la correlación entre el Atractivo Olfativo de las camisetas y el Atractivo Global de los estímulos fue más fuertemente significativa que la correlación entre el Atractivo Visual de las fotografías y el Atractivo Global. En la Tabla 2 se presentan los resultados de la regresión. Se excluyó la variable Atractivo Visual ya que no predijo significativamente el Atractivo Global.

Sólo el Atractivo Olfativo de las camisetas predijo significativamente el Atractivo Global. Esta variable explicó una porción importante de variancia del Atractivo Global $\left[R^{2}=.662, F(1,12)=23.459, p=.000\right]$.

\section{Resumen de LOS hallazgos}

El Atractivo Olfativo de las camisetas y el Atractivo Visual de las fotografías no están correlacionados en ninguno de los dos grupos. Sólo el Atractivo Olfativo de las camisetas difirió significativamente a partir de la orientación sexual de las personas que las usaron; tanto el Atractivo Visual de las fotografías como el Atractivo Olfativo de las camisetas predijeron el Atractivo Global de los sujetos heterosexuales, aunque el Atractivo Visual de las fotografías lo hizo de más significativamente, y sólo el Atractivo Olfativo de las camisetas predijo el Atractivo Global de los sujetos homosexuales.

\section{Discusión}

Las dos primeras hipótesis planteadas (que habría una correlación entre los punta- 
jes para Atractivo Olfativo y Atractivo Visual) resultaron desacertadas. Esta falta de correlación contradice hallazgos previos. Según lo reportado por Foster (2008) por ejemplo, el atractivo del olor corporal de hombres se correlaciona positivamente con su atractivo facial y negativamente con su grado de asimetría fluctuante. La asimetría funcional o asimetría fluctuante se refiere a cuando los dos hemisferios, que en teoría tienen funciones proporcionadas, se especializan más de lo normal, y cada uno lleva a cabo funciones ligeramente distintas. En teoría, esta asimetría es visible en el cuerpo (por ejemplo, longitud de los pies, tobillos, muñecas, codos, orejas y dedos).

La contradicción que se encontró puede deberse a que la mayoría de las investigaciones anteriores han consistido en reportes realizados por mujeres, que evaluaban el atractivo de estímulos provenientes de hombres. Hombres homosexuales realizaron los juicios de atractivo que se midieron en esta investigación. Ellos representan un grupo muy poco estudiado con respecto al papel de los sentidos en la elección de pareja. Es posible, entonces, que los mecanismos que operan en el caso de dicha población difieran de los observados con sujetos heterosexuales.

Por otro lado, Foster (2008) también sugiere que los resultados de trabajos anteriores sobre los sentidos en la elección de pareja podrían ser espurios debido al uso de auto-reportes. Su lógica es que a mayor distancia temporal entre la percepción de un estímulo y el reporte sobre su atractivo, la influencia de otros factores, como recuerdos o sentimientos, es mayor, lo cual distorsiona el resultado.

Podría ser que en anteriores trabajos, los sujetos hayan asociado el Atractivo Olfativo con el facial a partir de su memoria. En ese caso, la evaluación habría sido indirecta. En un trabajo de Cornwell y colaboradores (2004) se intentó corroborar empíricamente la hipótesis que planteaba una correlación entre los atractivos olfativo y facial. Se encontraron correlaciones positivas significativas entre rostros masculinos y una fero- mona masculina. Sin embargo, las correlaciones fueron débiles.

Es posible, a partir de lo que se ha discutido, que la forma de medir los constructos en el pasado explique las inconsistencias entre los estudios. Pareciera que, a medida que los métodos de evaluación se aproximan a la Psicofísica (medición de tiempos de respuesta, estímulos discretos, evaluaciones hechas al momento de la prueba), los hallazgos se asemejan más a lo que se encontró en la presente investigación.

Habría que considerar también que el procesamiento de información sensorial es distinto según el sentido del cual se trate. La información olfativa viaja en forma directa de los receptores olfativos, localizados en la mucosa nasal, a los bulbos olfatorios y de ahí, la información se proyecta al resto del cerebro (a la corteza olfativa y al tálamo dorsomedial) mediante los axones que componen los tractos olfativos (Carlson, 1996).

Por otro lado, la información visual hace un largo recorrido antes de llegar a la corteza estriada, donde se procesa finalmente en lo que se entiende como una imagen. Pasa primero por el tálamo (por donde también pasa la información auditiva), después por el hipotálamo y el tectum, estas son estructuras que además de regular los ciclos sueño-vigilia (la actividad durante el día y durante la noche), también influyen sobre la asociación de emociones con recuerdos basados en información visual (Carlson, 1996).

Lo anterior pudo haber influido sobre los hallazgos encontrados con esta investigación. Es posible que en la forma de procesamiento de la información visual, interfiera un mayor número de asociaciones, recuerdos y gustos construidos a lo largo del desarro1lo del individuo. El olfato, en vista de la comunicación más directa que tiene con la corteza cerebral, sin desviaciones previas a otras estructuras, podría transmitir información química como tal, sin las connotaciones afectivas con las que más probablemente se cargue la información visual.

Podría ser entonces, que el Atractivo Visual de las fotografías haya sido relacionado más con gustos personales de los evaluado- 
res que con el Atractivo Olfativo de las camisetas, ya que, de éstas últimas, se percibió información que viajó más directamente a la corteza cerebral.

Por otro lado, el olfato es un sentido mucho menos ejercitado culturalmente, por lo cual las preferencias olfativas de los sujetos podrían relacionarse más con cuestiones neurofisiológicas que sus preferencias visuales, las cuales podrían tener que ver con su memoria y con sus experiencias previas.

Según lo planteado hasta aquí, los resultados obtenidos hacen pensar en cierta contradicción con lo reportado por Foster (2008), que el atractivo facial de un sujeto podría no ser una buena señal de su calidad genética, al menos cuando quien evalúa el atractivo es un hombre homosexual.

De cualquier manera sería importante tener en cuenta el número de sujetos que participó en el experimento que se informa, ya que esto resulta un factor importante al pensar en la validez externa de las observaciones.

La hipótesis 3 también resultó desacertada: no hubo diferencias en los puntajes para Atractivo Visual entre los dos tipos de estímulo (homo y heterosexual). Respecto a lo anterior, ciertos estudios muestran que mientras el olor es de especial relevancia para los juicios de atractivo que hacen mujeres, la visión juega un papel más importante en las evaluaciones de ese tipo que hacen hombres (Herz \& Inzlicht, 2002; Sergeant et al., 2005). En otras palabras, la visión es más relevante que el olfato en la elección de pareja que hacen los hombres.

Los resultados del experimento que se informa llevan a pensar que la corteza orbitofrontal podría representar valores de recompensa para los rostros de parejas sexuales potenciales, sin importar la orientación sexual de las mismas (aunque esto es una inferencia, ya que no se usó el método de imágenes por resonancia magnética funcional). En este caso en particular, pareciera que los evaluadores (hombres homosexuales) respondieron de igual forma ante cualquier rostro de hombre, lo cual sugiere que no consiguieron distinguir (ni consciente ni inconscientemente) entre los estímulos a partir de la orientación sexual de los sujetos evaluados.

Según la literatura sobre el tema, la asimetría fluctuante puede percibirse en el cuerpo de los hombres. Uno de los indicadores de baja asimetría fluctuante son los rostros con rasgos más masculinos (Thornhill \& Gangestad, 2003). A partir de este planteo, los experimentadores esperaban encontrar diferencias en los puntajes de Atractivo Visual a partir de la orientación sexual de los sujetos evaluados, ya que el Atractivo Facial es teóricamente distinto, según la orientación sexual del individuo.

Lo anterior remite a la hipótesis neurohormonal de la orientación sexual, según la cual anomalías hormonales durante el desarrollo prenatal determinan la atracción hacia uno u otro sexo, sin importar el sexo de la persona que se esté gestando. La asimetría fluctuante, referida en el párrafo anterior, es una de las señales de estas anomalías hormonales durante el desarrollo (Mustanski et al., 2002).

Sin embargo, los hallazgos de este estudio contradijeron lo esperado por los investigadores. Pareciera que en realidad, la asimetría fluctuante quizá no es perceptible a simple vista en los rostros de hombres, o que así sucede exclusivamente cuando los evaluadores son hombres homosexuales.

A diferencia de lo que ocurrió con la hipótesis 3 , se comprobó la hipótesis 4: se observaron diferencias estadísticamente significativas con respecto al Atractivo Olfativo a partir de la orientación sexual de los sujetos evaluados.

Lo anterior, además de ser un hallazgo esperado, es quizá el resultado más interesante de la investigación. Concuerda con lo reportado por Martins y colaboradores (2005): que las mujeres y los hombres homosexuales podrían producir olores axilares que los distingan de los heterosexuales. Los hallazgos del estudio realizado apoyan la hipótesis anterior. Además, hacen pensar que los hombres homosexuales podrían percibir los olores humanos de forma distinta que los heterosexuales.

Estos mismos resultados sugieren que los hombres homosexuales pudieron distinguir 
(inconscientemente) la orientación sexual de los sujetos cuyo atractivo evaluaron. Savic, Berglund y Lindström (2005) encontraron en relación con dicho descubrimiento, un patrón de activación hipotalámica ante feromonas humanas distinto para diferentes orientaciones sexuales.

La hipótesis 6 (que el Atractivo Olfativo predeciría el Atractivo Global de los sujetos evaluados) también se comprobó y se relaciona íntimamente con la hipótesis 4 . Mientras que, en el caso de los evaluados heterosexuales, tanto el olfato como la visión contribuyeron a los puntajes de Atractivo Global, en el caso de los evaluados homosexuales contribuyó solamente el olfato.

Parece entonces, que cuando los evaluadores de atractivo son hombres homosexuales, las claves olfativas son más importantes que las visuales. Como no existe gran cantidad de bibliografía al respecto, los planteos teóricos de aquí dependerán de futuras réplicas de este experimento.

En cuanto a la hipótesis 7 (que el Atractivo Visual predeciría mejor que el olfativo, el Atractivo Global de los evaluados heterosexuales), la cual también se comprobó, se plantea la posibilidad de que, al no percibirse huellas olfativas homosexuales, el olor de los sujetos evaluados pasó a segundo plano, mientras que el Atractivo Visual de sus fotografías, asociado en mayor medida con los recuerdos y preferencias individuales de los evaluadores, se volvió más relevante. En otras palabras, podría pensarse que como no hubo información química relevante (que revelara a los sujetos evaluados como homosexuales) para los evaluadores, ellos recurrieron a su sentido más entrenado culturalmente (la vista) para evaluar el atractivo de los estímulos.

Lo anterior, una vez más, refuerza la idea de que las personas homosexuales (en este caso, específicamente los hombres) producen olores corporales que las distinguen de sus contrapartes heterosexuales (Martins et al., 2005). No existe suficiente literatura para dar cuenta de estos resultados. Por lo pronto, lo que puede hacerse es sugerir que se continúe la investigación por la línea del presente trabajo.
Por último, la hipótesis 5 no se comprobó: los puntajes para Atractivo Global no difirieron de forma significativa a partir de la orientación sexual de los sujetos evaluados.

Dicho resultado podría relacionarse con que los puntajes altos o bajos con respecto al Atractivo Olfativo, se acercaron a la media cuando se realizó la evaluación del Atractivo Olfativo en presencia de las fotografías que correspondían a cada olor (al evaluar el Atractivo Global).

Los investigadores observaron que durante la fase de evaluación del Atractivo Global, muchos de los evaluadores expresaron su descontento ante la fotografía correspondiente a un olor que antes (durante la fase de evaluación del Atractivo Olfativo) habían encontrado placentero. Se observó también, que los puntajes globales eran mucho menores o mucho mayores que los puntajes olfativos, en el caso de ambos tipos de estímulo (homo y heterosexual). Esto se relaciona con lo discutido sobre la visión, un sentido más entrenado culturalmente, que se vincula con recuerdos, preferencias personales y asociaciones emocionales (Carlson, 1996). En otras palabras, podría pensarse que, cuando la visión se combina con el olfato, el primer sentido altera la pureza de la información química y se confunde con cuestiones menos estrictamente sensoriales (en el sentido de información física que se percibe del medio y viaja a la corteza cerebral sin asociarse con emociones ni memoria).

Sin embargo, lo anterior es una inferencia ya que parte de las observaciones realizadas al momento del experimento y no de bibliografía científica al respecto (la cual, según la búsqueda de los investigadores, no existe como tal).

\section{LIMITACIONES}

A pesar de que ciertas hipótesis cruciales fueron comprobadas, cabría preguntarse si los hallazgos de la investigación realizada (obtenidos en un escenario artificial) funcionan de la misma forma en la vida real. Es 
decir, si los hombres homosexuales dan más peso al olfato o a la visión en su elección cotidiana de pareja. Puede apreciarse que el olfato da información a los hombres homosexuales sobre la orientación sexual de la pareja potencial, pero esto no responde si los sujetos se basan en dicha información para dirigir su conducta hacia las personas con las que se cruzan día a día. Más aún, está la pregunta sobre si el olor guarda una relación en cuanto a la satisfacción de pareja que hay entre hombres homosexuales. Garver-Apgar y colaboradores (2006) encontraron que, cuando las parejas no compartían alelos del antígeno humano de leucocitos (cuando no había atracción olfativa significativa), las mujeres reportaron sentirse insatisfechas sexualmente, mientras que los hombres no reportaron diferencias en cuanto a su satisfacción sexual ni de pareja. El hecho de que posiblemente, para los hombres homosexuales sea relevante el atractivo del olor de sus parejas, abre la pregunta sobre si el atractivo del olor, como se mencionó, se relaciona con la satisfacción sexual y de pareja. También tendría que considerarse, como sugieren las investigaciones de los autores citados, si las relaciones son a corto o a largo plazo. Sería interesante seguir la investigación por esa línea, quizá con los mismos sujetos que participaron en este estudio.

Por otro lado, los resultados de esta investigación han de tomarse con reserva con respecto al tamaño final de la muestra (14 sujetos), ya que los hallazgos, aunque significativos y congruentes con el marco teórico, podrían no ser generalizables. Más que pensarse como resultados concluyentes, podrían tomarse como una invitación a nuevas investigaciones que sigan ésta como modelo en el aspecto metodológico e implementen las correcciones sugeridas a continuación (entre ellas, el tamaño de la muestra).

En cuanto a los estímulos, el hecho de que no se pudo controlar por completo que los participantes siguieran todas las instrucciones con respecto al uso de las camisetas pudo haber mermado la pureza de los olores corporales que se usaron como estímulos, así como el efecto que éstos tuvieron sobre los evaluadores. Algunos aspectos que pudieron haber interferido fueron: el tiempo durante el que usaron las camisetas, sólo dos noches, (en otras investigaciones se usaron al menos durante una semana previa a la evaluación), la alimentación y el consumo de alcohol, tabaco y otras sustancias que influyen en el aroma corporal. Aunque se les indicó a los sujetos no comer ciertos alimentos, no fumar, no beber, ni dormir con alguien más mientras utilizaran la camiseta, otras investigaciones han desplegado un mayor grado de control de estos factores. En ellas, los investigadores pedían por ejemplo, que las instrucciones se siguieran durante todo el día, y no solamente durante los lapsos cuando tuvieran puestas las camisetas. En cuanto a los estímulos visuales (fotografías), no hay limitantes metodológicas perceptibles por parte de los investigadores.

En cuanto a la administración del experimento, hubo ciertas condiciones que pueden atenderse en futuras investigaciones, como el espacio disponible para la presentación de los estímulos (sólo contábamos con cinco cubículos). Ésto implicó dividir cada fase en dos, lo cual dio oportunidad a los evaluadores para comentar los resultados con otros evaluadores entre fases e inclusive, de consolidar ciertos recuerdos olfativos que pudieron haber influido en el resto de las evaluaciones.

Un aspecto más es que posiblemente los evaluadores no percibieran el olor de las camisetas, pues hubo varios que comentaron no poder oler aroma alguno y fueron más activos en su evaluación, esto es, buscaron cómo sacar la camiseta de la bolsa para olerla mejor, actitud que muchos evaluadores no adoptaron a pesar de que posiblemente, no percibieron aroma alguno. Lo anterior pudo influir sobre las calificaciones.

Por último, hubiera sido conveniente que todos los sujetos evaluaran los estímulos sin que pasara tanto tiempo entre el primer grupo que evaluó y el último, ya que posiblemente, las cualidades de los estímulos olfativos variaron durante el tiempo transcurrido entre dichas evaluaciones. 
Teniendo en cuenta estas limitaciones, sería importante realizar una nueva investigación si se desea generalizar los resultados encontrados y poder incluirlos en futuros estudios.

\section{CONCLUSIONES}

Los resultados de esta investigación podrían contribuir valiosamente al debate actual con respecto a si existen feromonas humanas como tal (Pause, 2004), ya que permiten inferir que, de hecho, el olor corporal de parejas potenciales modifica el comportamiento de los seres humanos.

Por otro lado, la implicancia teórica más apremiante es que las personas homosexuales podrían funcionar de forma neuro-fisiológicamente distinta a las heterosexuales, al menos con respecto al papel que juega la senso-percepción en la elección de pareja que hacen.

Con respecto a la práctica, la realización de este experimento sugiere la recomendación de hacer diseños flexibles que se adapten a la población objetivo pues, en vista de la realidad social mexicana (es poco probable que pueda pagarse a los participantes), uno depende de la ayuda voluntaria de los sujetos y debe adaptarse a su disponibilidad lo más que se pueda.

Sería conveniente además, no presentar una serie larga de estímulos olfativos sin pausas, ya que esto podría aturdir al evaluador del atractivo. Por otro lado, no dejar pasar tanto tiempo entre el uso de las camisetas por los sujetos evaluados y la aplicación del experimento.

En la práctica, los hallazgos sobre diferencias biológicas entre personas homo y heterosexuales deberían ser tenidos en cuenta, para fomentar el respeto a la diversidad sexual. Es crucial enfatizar que la orientación sexual es un constructo multifactorial, imposible de reducir a explicaciones simplistas, así como que tanto la homo como la heterosexualidad requieren explicaciones científicas, más allá de su grado de adecuación a la norma. Debe problematizarse la orientación sexual en general, no sólo las orientaciones sexuales que se desvían del promedio.

Por otro lado, es importante resaltar que la orientación sexual es muy probablemente, el resultado tanto de factores orgánicos como de crianza y de la interacción entre ambos. No representa meramente una decisión personal ni una enfermedad. En México, aún se piensa de esa forma en ciertos sectores.

El conocimiento científico sobre el tema podrá usarse para fortalecer a una población vulnerable, cuyos derechos siguen sin respetarse del todo, mediante el argumento de que la orientación sexual podría ser inclusive, una configuración neurológica particular. 
Visión y olfato en las evaluaciones de atractivo

TABLA 1

ANÁLISIS DE REGRESIÓN DE LAS VARIABLES QUE PRONOSTICAN EL ATRACTIVO GLOBAL DE LOS SUJETOS HETEROSEXUALES

\begin{tabular}{|c|c|c|c|}
\hline Variables & B & EE & $\beta$ \\
\hline Atractivo visual de los estímulos heterosexuales & .659 & .195 & $.593^{\star *}$ \\
Atractivo olfativo de los estímulos heterosexuales & .490 & .193 & $.446^{\star *}$ \\
\hline
\end{tabular}

${ }^{*} p<.05$

${ }^{* *} p<.01$

TABLA 2

ANÁLISIS DE REGRESIÓN DE LAS VARIABLES QUE PRONOSTICAN EL ATRACTIVO GLOBAL DE LOS SUJETOS HOMOSEXUALES

\begin{tabular}{|c|c|c|c|}
\hline Variable & B & EE & $\beta$ \\
\hline Atractivo olfativo de los estímulos homosexuales & .85 & .175 & $.813^{* *}$ \\
\hline
\end{tabular}

${ }^{* *} p<.000$ 


\section{REFERENCIAS BIBLIOGRÁFICAS}

Carlson, N. (1996). Fundamentos de psicología fisiológica [Foundations of physiological psychology]. México: Prentice Hall.

Cerda, A.L., Mondragón, R. \& Díaz, V. (2000). Comunicación química en primates [Chemical communiction in primates]. Salud Mental, 23, 25-32.

Cornwell, R.E., Boothroyd, L., Burt, D.M., Feinberg, D. R., Jones, B.C., Little, A.C. et al. (2004). Concordant preferences for oppositesex signals? Human pheromones and facial characteristics. Proceedings of the Royal Society B: Biological Sciences, 271, 635-640. doi: 10.1098/rspb.2003.2649.

Chaix, R., Cao, C. \& Donnelly, P. (2008). Is mate choice in humans MHC-dependent? Plos Genetics, 4(9). doi:10.1371/journal.pgen.10 001 84.

DeBruine, L.M., Jones, B.C., Unger, L., Little, A.C. \& Feinberg, D.R. (2007). Dissociating averageness and attractiveness: Attractive faces are not always average. Journal of Experimental Psychology: Human Perception and Performance, 33, 1420-1430. doi: 10.10 37/0096-1523.33.6.1420.

Foster, J.D. (2008). Beauty is mostly in the eye of the beholder: Olfactory versus visual cues of attractiveness. Journal of Social Psychology, 148(6), 765-774. doi: 10.32 00/SOC P.14 8.6. 765-774.

Garver-Apgar, C.E., Gangestad, S.W., Thornhill, R., Miller, R. \& Olp, J.J. (2006). Major histocompatibility complex alleles, sexual responsivity, and unfaithfulness in romantic couples. Psychological Science, 17, 830-835. doi: 10.1 111/j.1467-9280.2006.01789.x.

Gobrogge, K.L., Perkins, P.S., Baker, J.H., Balcer, K.D., Breedlove, S.M. \& Klump, K.L. (2007). Homosexual mating preferences from an evolutionary perspective: Sexual selection theory revisited. Archives of Sexual Behavior,
36, 717-723. doi: 10.1007/s10508-007-9216$\mathrm{x}$.

Grammer, K., Fink, B. \& Neave, N. (2005). Human pheromones and sexual attraction. European Journal of Obstetrics \& Gynecology and Reproductive Biology, 118, 135-142. doi: 10.1 016/j.ejogrb.2004.08.010.

Havlíček, J., Dvořákov, R., Barto, L. \& Flegr, J. (2006). Non-advertized does not mean concealed: Body odour changes across the human menstrual cycle. Ethology, 112(1), 81-90. doi: 10.1111/j.1439-0310.2006.01125.x.

Herz, R.S. \& Inzlicht, M. (2002). Sex differences in response to physical and social factors involved in human mate selection. The importance of smell for women. Evolution and Human Behavior, 23, 359-364. doi: 10.1016/ S1090-5138(02)00095-8.

Jacob, S., McClintock, M.K., Zelano, B. \& Ober, C. (2002). Paternally inherited HLA alleles are associated with women's choice of male odor. Nature Genetics, 30(2), 175-179. doi: 10.1038/ng830.

Kinsey, A.C., Pomeroy, W.B. \& Martin, C.E. (1948). Sexual behavior in the human male. Philadelphia: W.B. Saunders.

Kranz, F. \& Ishai, A. (2006). Face perception is modulated by sexual preference. Current Biology, 16, 63-68. doi:10.1016/ j.cub.2005. 10.070 .

Levin, R.F. (2004). Smells and tases - their putative influence on sexual activity in humans. Sexual and Relationship Therapy, 19(4), 451462. doi: 10.1080/14681990412331315135.

Martins, Y., Preti, G., Crabtree, C.R., Runyan, T., Vainius, A.A. \& Wysocki, C.J. (2005). Preference for human body odors is influenced by gender and sexual orientation. Psychological Science, 16(9), 694-701. doi:10.1111/j.146792 80.2005.01598.x.

McBurney, D.H., Shoup, M.L. \& Streeter, S.A. (2006). Olfactory comfort: Smelling a partner's clothing during periods of separation. 
Journal of Applied Social Psychology, 36, 2325-2335. doi: 10.1111/j.0021-9029.2006. 00105.x.

Mustanski, B.S., Chivers, M.L. \& Bailey, J.M. (2002). A critical review of recent biological research on human sexual orientation. Annual Review of Sex Research, 13, 89-140. doi: 10. $1177 / 0146167210378755$.

Pause, B. (2004). Is the human skin a pheromoneproducing organ? Journal of Cosmetic Dermatology, 3, 223-228. doi:10.1111/j.1473213 0.2004.00106.x.

Rennels, J.L., Bronstad, P.M. \& Langlois, J.H. (2008). Are attractive men's faces masculine or feminine? the importance of type of facial stimuli. Journal of Experimental Psychology, 34(3), 884-893. doi:10.1037/0096-1523.34.4. 884.

Roberts, S.C., Havlicek, J., Flegr, J., Hruskova, M., Little, A.C., Jones, B.C. et al. (2004). Female facial attractiveness increases during the fertile phase of the menstrual cycle. Proceedings of the Royal Society B: Biological Sciences, 271, 270-272. doi:10.1098/ rsbl.2004.0174.
Savic, I., Berglund, H. \& Lindström, P. (2005). Brain response to putative pheromones in homosexual men. Proceedings of the National Academy of Sciences, 102, 7356-7361. doi: 10.1073/pnas.0407998102.

Sergeant, M.J.T., Davies, M.N.O., Dickins, T.E. \& Griffiths, M.D. (2005). The self-reported importance of olfaction during human mate choice. Sexualities, Evolution \& Gender, 7 , 199-213. doi:10.1080/14616660500173685.

Sergeant, M.J.T., Dickins, T.E., Davies, M.N.O. \& Griffiths, M.D. (2007). Women's hedonic ratings of body odor of heterosexual and homosexual men. Archives of Sexual Behavior, 36, 395-401. doi:10.1007/s10508-006-91263.

Shoup, M.L., Streeter, S.A. \& McBurney, D.H. (2008). Olfactory comfort and attachment within relationships. Journal of Applied Social Psychology, 38, 2954-2963. doi:10.1111/ j.1559-1816.2008.00420.x.

Thornhill, R. \& Gangestad, S.W. (2003). Evolutionary theory led to evidence for a male sex pheromone that signals symmetry. Psychological Inquiry, 14(3/4), 318-325. doi: 10.10 80/1047840X.2003.9682899.

Universidad Iberoamericana (UIA) México D.F. México

Fecha de recepción: 18 de abril de 2012 Fecha de aceptación: 7 de enero de 2013 
